\title{
Alectinib can replace crizotinib as standard first-line therapy for $A L K$-positive lung cancer
}

\author{
Takehiro Uemura, Toyoaki Hida
}

Department of Thoracic Oncology, Aichi Cancer Center Hospital, Nagoya, Japan

Correspondence to: Toyoaki Hida, MD, PhD. Department of Thoracic Oncology, Aichi Cancer Center Hospital, 1-1 Kanokoden, Chikusa-ku, Nagoya, Aichi 464-8681, Japan. Email: 107974@aichi-cc.jp.

Provenance: This is a Guest Editorial commissioned by Section Editor Jianrong Zhang, MD (George Warren Brown School of Social Work, Washington University in St. Louis, St. Louis, USA).

Comment on: Peters S, Camidge DR, Shaw AT, et al. Alectinib versus crizotinib in untreated ALK-positive non-small-cell lung cancer. N Engl J Med 2017;377:829-38.

Submitted Aug 16, 2017. Accepted for publication Aug 22, 2017.

doi: $10.21037 /$ atm.2017.08.36

View this article at: http://dx.doi.org/10.21037/atm.2017.08.36

In 2007, Soda and colleagues identified anaplastic lymphoma kinase $(A L K)$ rearrangement in adenocarcinoma of the lung (1). Approximately $5 \%$ of non-small cell lung cancer (NSCLC) cases have $A L K$ rearrangement and the incidence of $A L K$-positive NSCLC is similar across all regions of the world. For patients with $A L K$-positive NSCLC, crizotinib (Xalkori; Pfizer), which is an $A L K$ targeted tyrosine kinase inhibitor (TKI), had been the standard first-line therapy that enabled patients to achieve prolonged progression-free survival (PFS) (2-5). However, treatment of $A L K$-positive NSCLC with crizotinib has some problems. First, most patients relapse within a year of treatment due to a variety of resistance mechanisms. Second, crizotinib is generally well tolerated; however, a number of significant toxicities, like gastrointestinal side effects, may occur sometimes and require dose modification or treatment discontinuation $(4,5)$. Third, crizotinib has poor penetration to the central nervous system (CNS), which is a common site for metastasis in $A L K$-positive NSCLC patients (6-8). Therefore, there has been a need for new and improved alternative agents to crizotinib.

In the June 2017 issue of the New England Fournal of Medicine, Peters and colleagues showed the superiority of alectinib to crizotinib in terms of efficacy and lower toxicity in a randomized, open-label, phase III trial for primary treatment of $A L K$-positive NSCLC (9). This study, namely the ALEX trial, compared as primary endpoint the investigator-assessed PFS rates between oral alectinib
(Alecensa; Genentech) at a dose of $600 \mathrm{mg}$ twice daily and oral crizotinib at a dose of $250 \mathrm{mg}$ twice daily. Secondary endpoints were PFS assessed independently by a review committee, time to CNS progression, objective response rate (ORR), and overall survival (OS). Eligible patients had histologically or cytologically confirmed advanced NSCLC that was $A L K$-positive by VENTANA ALK (D5F3) immunohistochemical assay (Ventana Medical Systems, Inc., Tucson, AZ, USA). Patients who had no previous systemic treatment for advanced NSCLC were eligible in this study. The 12-month, event-free rate of investigatorassessed PFS was significantly higher with alectinib [68.4\%, $95 \%$ confidence interval (CI): 61.0-75.9\%] than with crizotinib (48.7\%, 95\% CI: 40.4-56.9\%). The median PFS with alectinib was not reached [95\% CI: 17.7 months-not estimable (NE)], compared with 11.1 months (95\% CI: 9.1-13.1) with crizotinib. Independent review committeeassessed PFS was significantly longer with alectinib (25.7 months, 95\% CI: 19.9-not estimable) than with crizotinib (10.4 months, 95\% CI: 7.7-14.6) [hazard ratio (HR), 0.50; 95\% CI: $0.36-0.70 ; \mathrm{P}<0.001]$. The ORR was higher with alectinib (82.9\%; 95\% CI: 76.0-88.5\%) than with crizotinib (75.5\%; 95\% CI: 67.8-82.1\%). The OS rates for both agents are currently immature. The time to CNS progression was significantly longer with alectinib than with crizotinib (cause-specific HR, 0.16; 95\% CI: 0.10-0.28, $\mathrm{P}<0.001)$. The 12 -month cumulative incidence rate of CNS progression was $9.4 \%$ (95\% CI, $5.4-14.7 \%$ ) versus $41.4 \%$ 
(95\% CI, 33.2-49.4\%). The adverse events that were more common in crizotinib than alectinib included nausea $(48 \%$ vs. $14 \%$ ), diarrhea ( $45 \%$ vs. $12 \%)$, and vomiting (38\% vs. $7 \%)$. The adverse events that occurred at a higher incidence with alectinib than with crizotinib were anemia $(20 \% \mathrm{vs}$. $5 \%$ ), myalgia (16\% vs. $2 \%$ ), increased blood bilirubin $(15 \%$ vs. $1 \%$ ), weight gain (10\% vs. $0 \%)$, musculoskeletal pain (7\% vs. $2 \%$ ), and photosensitivity reaction ( $5 \%$ vs. $0 \%$ ). Grade 3 to 5 adverse events occurred in $50 \%$ of the patients treated with crizotinib and in $41 \%$ of the patients treated with alectinib. The rate of adverse events leading to dose reduction, interruption, or discontinuation was lower with alectinib than with crizotinib. From these results, alectinib had longer PFS, lower CNS progression rate, and lower toxicity compared with crizotinib.

While the ALEX trial was successfully achieved, the J-ALEX trial, which is a similar randomized phase III trial on alectinib versus crizotinib for ALK inhibitor-naive Japanese patients with $A L K$-positive NSCLC, had already been reported in May 2017 (10). This trial compared as primary endpoint the PFS rates assessed by an independent review facility (IRF) between oral alectinib at a dose of $300 \mathrm{mg}$ twice daily and oral crizotinib at a dose of $250 \mathrm{mg}$ twice daily. The secondary endpoints were investigatorassessed PFS, ORR, OS, duration of response, time to response, time to CNS progression, health-related quality of life, safety, and pharmacokinetics. Although patients who had no previous systemic treatment were eligible in ALEX trial, those who were chemotherapy-naïve or had received one previous chemotherapy regimen were eligible in the J-ALEX trial. The diagnosis of $A L K$ positivity was confirmed by immunohistochemistry and fluorescence in situ hybridization (FISH) or reverse transcription polymerase chain reaction (RT-PCR) using tissue or cell samples. This study, on its second preplanned interim analysis, met its primary endpoint of showing superiority of alectinib over crizotinib in IRF-assessed PFS. The median PFS significantly improved with alectinib (NE, 95\% CI: 20.3 months-NE) compared with that with crizotinib (10.2 months, 95\% CI: 8.2-12.0; HR, 0.34; 99.7\% CI: $0.17-0.71)$. The ORR assessed by the IRF was greater with alectinib (92\%, 95\% CI: 85.6-97.5\%) than with crizotinib (79\%, 95\% CI: 70.5-87.3\%); the ORR assessed by the investigators also favored alectinib (85\%, 95\% CI: 78.6-92.3\%) over crizotinib (70\%, 95\% CI: 61.4-79.0\%). The OS rates in both groups are still immature. The total number of patients with at least one grade 3 or 4 adverse event was higher in the crizotinib group (52\%) than in the alectinib group (26\%).

The differences in the study criteria, baseline patient characteristics, and results between the ALEX and J-ALEX trials are summarized in Tables 1 and 2. Only Japanese patients were enrolled in J-ALEX trial, whereas patients from various countries were enrolled in the ALEX trial, which was an international cohort study. Furthermore, the $600 \mathrm{mg}$ twice daily dose of alectinib was administered as the global standard in the ALEX trial, compared with the $300 \mathrm{mg}$ twice daily administration of alectinib in the J-ALEX trial. Despite some of these differences, the results of two studies had extreme resemblance. At this time, the results of the J-ALEX study were confirmed by the results of the ALEX study.

Following the ALEX trial, first-line administration of alectinib leads to better PFS than that of crizotinib. Our next question would be on the choice between crizotinib and alectinib as first-line agent to prolong OS. The order in which crizotinib and alectinib should be prescribed in order to achieve maximum benefit to patients is unclear. Consensus on this matter must be confirmed by the OS rates of the patients in the ALEX and J-ALEX trials, although there were some reports on sequential administration of crizotinib and alectinib. Sequential treatment with crizotinib followed by alectinib has been reported to produce a combined PFS of 18.2 months in a small study (11) and another report showed that alectinib administration after crizotinib failure tended to provide a better OS benefit than did therapy with alectinib alone in patients with $A L K$-positive NSCLC (12). In ALEX, the PFS of alectinib assessed by investigators has not been reached and that assessed by the independent review committee was 25.7 months. A simple comparison of these results showed that upfront administration of alectinib appeared to translate into greater efficacy than sequential treatment with crizotinib and alectinib. Following the PFS of the ALEX trial, it would be no overstatement to say that alectinib can replace crizotinib as standard first-line therapy for $A L K$-positive lung cancer.

The efficacy and feasibility of alectinib for patients with poor performance status (PS) are also unknown. In the ALEX trial, only patients classified as Eastern Cooperative Oncology Group (ECOG) PS 0-2 could be enrolled; therefore, the outcomes in patients with poor PS is uncertain. A small sample size-study has reported that the PFS of advanced $A L K$-positive NSCLC patients with PS of 2 to 4 who received alectinib orally at $300 \mathrm{mg}$ twice daily was 16.2 months (13). In the ALEX study, the adverse events in the alectinib group were milder than those in the 
Table 1 Baseline patient characteristics of ALEX and J-ALEX trial

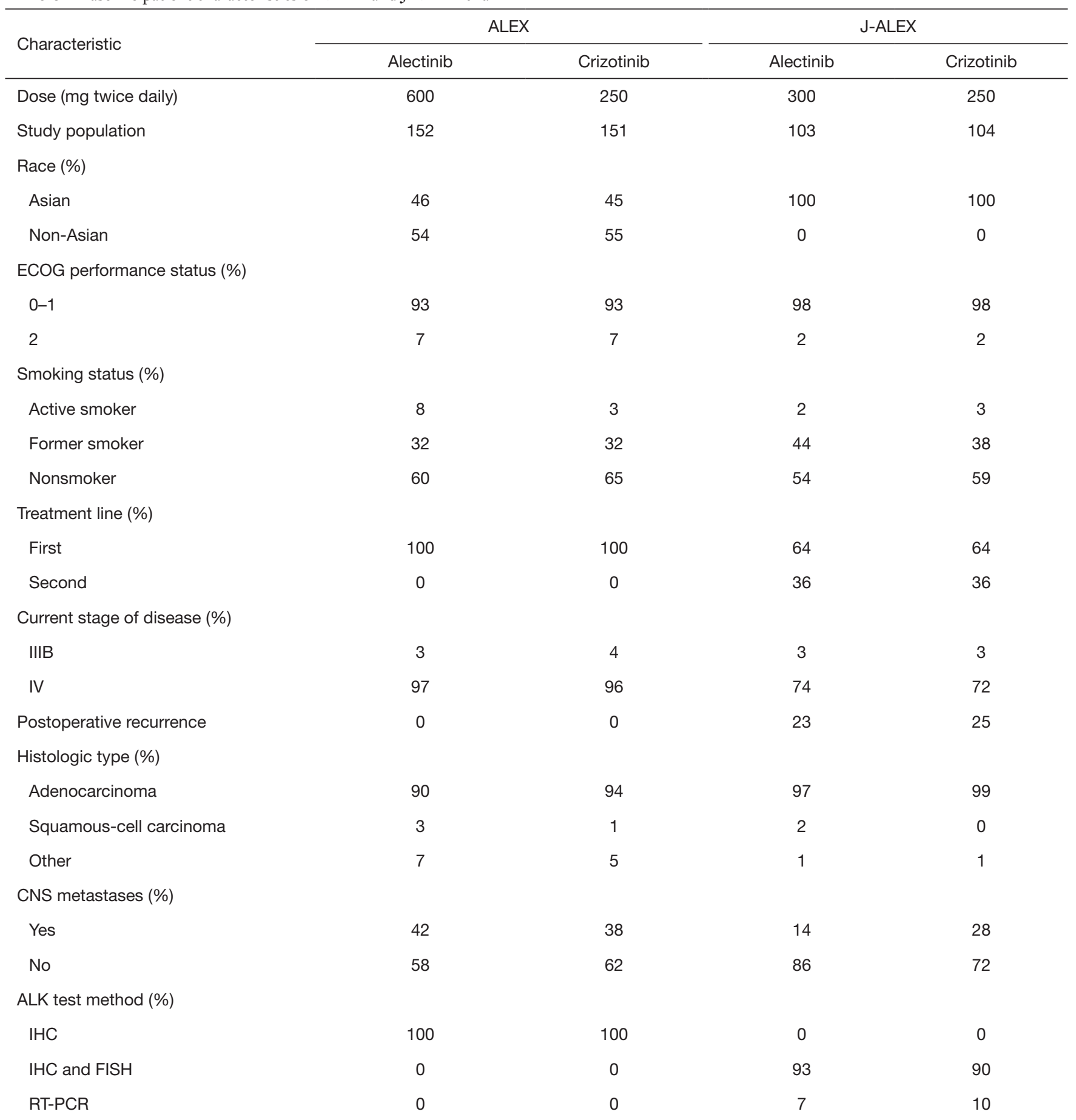

ECOG, Eastern Cooperative Oncology Group; CNS, central nervous system; ALK, anaplastic lymphoma kinase; FISH, fluorescence in situ hybridization; RT-PCR, reverse transcription polymerase chain reaction. 
Table 2 PFS and response in ALEX and J-ALEX

\begin{tabular}{|c|c|c|c|c|}
\hline Variable & \multicolumn{2}{|c|}{ ALEX } & \multicolumn{2}{|c|}{ J-ALEX } \\
\hline Investigator assessed PFS (months) & Not reached & 11.1 & & \\
\hline Independent review committee-assessed PFS (months) & 25.7 & 10.4 & Not reached & 10.2 \\
\hline \multicolumn{5}{|l|}{ Response (investigator-assessed) } \\
\hline Complete response (\%) & 4 & 1 & 5 & 2 \\
\hline Partial response (\%) & 79 & 74 & 81 & 68 \\
\hline Stable disease (\%) & 16 & 16 & 13 & 18 \\
\hline Median duration of response (months) & NE & 11.1 & NE & 11.2 \\
\hline
\end{tabular}

PFS, progression free survival; NE, not estimable.

crizotinib group, suggesting that alectinib may be a more suitable treatment option than crizotinib in patients with poor PS. The efficacy and feasibility of alectinib treatment in patients with $A L K$ rearrangement-positive NSCLC and a poor PS should be confirmed in a future trial with larger sample size.

For the treatment of $A L K$-positive lung cancer, the Food and Drug Administration (FDA) has now approved four ALK-TKI drugs, including crizotinib as first generation and alectinib, ceritinib (Zykadia; Novartis), and brigatinib (Alunbrig; Ariad/Takeda) as second generation. The drugs that are currently under investigation include: (I) entrectinib (RXDX-101) and ensartinib (X-396), which are TKIs that have multiple molecular targets, including $A L K$; and (II) lorlatinib, the next third generation agent.

At present, the superiority of alectinib over crizotinib as first-line treatment for $A L K$-positive NSCLC was proven by the ALEX trial; however, the choice among alectinib, ceritinib, brigatinib, and lorlatinib as first-line treatment is uncertain. First-line ceritinib showed a statistically significant and clinically meaningful improvement in PFS compared with chemotherapy in patients with advanced ALK-rearranged NSCLC (ASCEND-4) (14). Brigatinib versus crizotinib (ALTA-1L; NCT02737501), lorlatinib versus crizotinib (NCT03052608), and ensartinib versus crizotinib (eXalt3; NCT02767804) in advanced $A L K$ rearranged NSCLC as a phase III study are now underway. If the superiority of these drugs over crizotinib as first-line treatment for $A L K$-positive NSCLC is proven, the ALK inhibitor that should be administered as first-line treatment must be confirmed by further head-to-head trial in the future.

Resistance to alectinib is another important problem that certainly appeared despite the extremely potent efficacy of alectinib. It is necessary to discover the method for conquering resistance to ALK inhibitors. The wellknown mechanisms of resistance to ALK-TKIs include: (I) $A L K$-dependent, "on-target" mechanisms, including $A L K$ secondary resistance mutations or amplification, where the tumor cell dependency on $A L K$ signaling persists; and (II) $A L K$-independent, "off-target" mechanisms, including activation of bypass signaling pathways, lineage changes, and drug efflux pump (15). Evaluation of patients who have relapsed on one next-generation ALK inhibitor and matching them to the next-best agent, based on secondary $A L K$-resistant status, will eventually resolve this outstanding problem. In a report on $17 A L K$-positive patients who underwent repeat biopsies following progression on alectinib (16), $A L K$ resistance mutations were found in $9(53 \%)$ specimens. In this report, same $A L K$ resistance mutations following progression on crizotinib were seen in 11/55 (20\%). The most common $A L K$ resistance mutation following progression on alectinib was G1202R (29\%), which resulted in much higher degrees of resistance to all currently available first- and second-generation ALK inhibitors. The other $A L K$ resistance mutations identified within the complete alectinib-resistant cohort included I1171N/S/T (12\%), V1180L (6\%), and L1196M (6\%). Tailoring of $A L K$ therapy after failure on alectinib must, likewise, be important in the small proportion of cases with uncommon and refractory mutations, such as $A L K$ G1202R. The third-generation pan-inhibitory ALK-TKI 
lorlatinib has been shown to effectively inhibit G1202R in cell lines and in patients $(15,17)$ and that it could be an option for patients pretreated with alectinib. Combination approaches targeting other pathways that mediate $A L K$ resistance may be needed to suppress the emergence of other important resistance mechanisms categorized in $A L K$ independent, bypass signaling track, particularly, EGFR and HER2 activation and MET amplification. Repeat biopsies to identify $A L K$ resistance mutations will play a larger role in guiding therapy decisions and further research in these cases after progression on alectinib. While the mechanism of resistance to ALK inhibitors in $A L K$-positive NSCLC is gradually clarified, the determinants of sensitivity to ALK inhibitors are also becoming clearer. Few studies have shown the therapeutic efficacy of crizotinib according to different variants in patients with $A L K$-positive NSCLC (18-20). Furthermore, the association between the $A L K$ variant and the frequency of $A L K$ resistance mutation status was also reported (21). Knowledge of the $A L K$ variants may help predict the sensitivity and resistance mechanism to ALK-TKI, including alectinib.

In conclusion, the findings of the ALEX trial revealed that alectinib is more suitable than crizotinib as the standard first-line therapy for $A L K$-positive lung cancer in terms of efficacy, toxicity, and prevention of CNS metastases. However, some important problems remain to be addressed; these include the most suitable among the second and third generation ALK inhibitors for first-line therapy and the strategies to overcome resistance to alectinib. A continuous study about the best sequence of ALK-TKI treatment and overcoming resistance will solve these problems in the future.

\section{Acknowledgements}

None.

\section{Footnote}

Conflicts of Interest: T Hida has received grants and personal fees from AstraZeneca, Ono Pharmaceutical Co., Ltd., Chugai Pharmaceutical Co., Ltd., Eli Lilly, Novartis, Taiho Pharmaceutical Co., Ltd., Nippon Boehringer Ingelheim, Pfizer, Bristol-Meyers Squibb, Clovis Oncology, MSD, and Kissei; grants from Eisai, Takeda Pharmaceutical Co., Ltd., Dainippon Sumitomo Pharma, Abbvie, Merck Serono, Kyowa Hakko Kirin, Daiichi Sankyo, Astellas, Ignyta, and Servier. T Uemura has no conflicts of interest to declare.

\section{References}

1. Soda M, Choi YL, Enomoto M, et al. Identification of the transforming EML4-ALK fusion gene in non-small-cell lung cancer. Nature 2007;448:561-6.

2. Camidge DR, Bang YJ, Kwak EL, et al. Activity and safety of crizotinib in patients with ALK-positive non-small-cell lung cancer: updated results from a phase 1 study. Lancet Oncol 2012;13:1011-9.

3. Kwak EL, Bang YJ, Camidge DR, et al. Anaplastic lymphoma kinase inhibition in non-small-cell lung cancer. N Engl J Med 2010;363:1693-703.

4. Shaw AT, Kim DW, Nakagawa K, et al. Crizotinib versus chemotherapy in advanced ALK-positive lung cancer. $\mathrm{N}$ Engl J Med 2013;368:2385-94.

5. Solomon BJ, Mok T, Kim DW, et al. First-line crizotinib versus chemotherapy in ALK-positive lung cancer. N Engl J Med 2014;371:2167-77.

6. Costa DB, Kobayashi S, Pandya SS, et al. CSF concentration of the anaplastic lymphoma kinase inhibitor crizotinib. J Clin Oncol 2011;29:e443-5.

7. Johung KL, Yeh N, Desai NB, et al. Extended survival and prognostic factors for patients with ALK-rearranged nonsmall-cell lung cancer and brain metastasis. J Clin Oncol 2016;34:123-9.

8. Yoshida T, Oya Y, Tanaka K, et al. Clinical impact of crizotinib on central nervous system progression in ALKpositive non-small lung cancer. Lung Cancer 2016;97:43-7.

9. Peters S, Camidge DR, Shaw AT, et al. Alectinib versus crizotinib in untreated ALK-positive non-small-cell lung cancer. N Engl J Med 2017;377:829-38.

10. Hida T, Nokihara H, Kondo M, et al. Alectinib versus crizotinib in patients with ALK-positive non-small-cell lung cancer (J-ALEX): an open-label, randomised phase 3 trial. Lancet 2017;390:29-39.

11. Watanabe S, Hayashi H, Okamoto K, et al. Progressionfree and overall survival of patients with ALK rearrangement-positive non-small cell lung cancer treated sequentially with crizotinib and alectinib. Clin Lung Cancer 2016;17:528-34.

12. Ito K, Hataji O, Kobayashi H, et al. Sequential therapy with crizotinib and alectinib in ALK-rearranged nonsmall cell lung cancer-A multicenter retrospective study. J Thorac Oncol 2017;12:390-6.

13. Iwama E, Goto Y, Murakami H, et al. Alectinib for patients with ALK rearrangement-positive non-small cell lung cancer and a poor performance status (Lung Oncology Group in Kyushu 1401). J Thorac Oncol 2017;12:1161-6. 
14. Soria JC, Tan DS, Chiari R, et al. First-line ceritinib versus platinum-based chemotherapy in advanced ALKrearranged non-small-cell lung cancer (ASCEND-4): a randomised, open-label, phase 3 study. Lancet 2017;389:917-29.

15. Lin JJ, Riely GJ, Shaw AT. Targeting ALK: Precision medicine takes on drug resistance. Cancer Discov 2017;7:137-55.

16. Gainor JF, Dardaei L, Yoda S, et al. Molecular mechanisms of resistance to first- and second-generation ALK inhibitors in ALK-rearranged lung cancer. Cancer Discov 2016;6:1118-33.

17. Zou HY, Friboulet L, Kodack DP, et al. PF-06463922, an ALK/ROS1 inhibitor, overcomes resistance to first and second generation ALK inhibitors in preclinical models. Cancer Cell 2015;28:70-81.

Cite this article as: Uemura T, Hida T. Alectinib can replace crizotinib as standard first-line therapy for $A L K$-positive lung cancer. Ann Transl Med 2017;5(21):433. doi: 10.21037/ atm.2017.08.36
18. Heuckmann JM, Balke-Want H, Malchers F, et al. Differential protein stability and ALK inhibitor sensitivity of EML4-ALK fusion variants. Clin Cancer Res 2012;18:4682-90.

19. Lei YY, Yang JJ, Zhang XC, et al. Anaplastic lymphoma kinase variants and the percentage of ALK-positive tumor cells and the efficacy of crizotinib in advanced NSCLC. Clin Lung Cancer 2016;17:223-31.

20. Yoshida T, Oya Y, Tanaka K, et al. Differential crizotinib response duration among ALK fusion variants in ALKpositive non-small-cell lung cancer. J Clin Oncol 2016;34:3383-9.

21. Ou SI. Association of ALK resistance mutations by EML4ALK variant (v3 vs. non-v3) in ALK+ non-small cell lung cancer (NSCLC). J Clin Oncol 2017;35:abstr 9010. 